\title{
Strategische Kultur und Sicherheitsstrategien in Deutschland
}

\author{
Christopher Daase und Julian Junk*
}

\begin{abstract}
Strategic culture has traditionally been analyzed as the capability of political elites and the strategic community. This article argues that it is important to incorporate the values and attitudes of society in order to grasp the inconsistencies and discrepancies between societal demands for security and national and international capabilities to supply it. The article analyzes accordingly the current state of the German foreign and security policy.
\end{abstract}

Keywords: Strategic culture, foreign and security policy, Germany

Strategische Kultur, Außen- und Sicherheitspolitik, Deutschland

\section{Einleitung ${ }^{1}$}

$\mathrm{D}$ ie Entscheidung der deutschen Bundesregierung, sich am 17. März 2011 bei der Abstimmung im UN-Sicherheitsrat über die Resolution 1973 zur Mandatierung einer internationalen Intervention in Libyen zu enthalten, führte einmal mehr zu einer kontroversen Diskussion über die strategischen Prioritäten Deutschlands in der Außen- und Sicherheitspolitik. Dabei hatte die Bundesregierung große Mühe, ihre Entscheidung, nicht mit den westlichen Bündnispartnern Frankreich, Großbritannien und den USA zu stimmen, zu rechtfertigen und Zweifel, die im transatlantischen Bündnis, aber auch in der deutschen Öffentlichkeit an der Berechenbarkeit und Verlässlichkeit Deutschlands aufgekommen waren, zu zerstreuen. Auch in der wissenschaftlichen Debatte ist das Abstimmungsverhalten im Fall Libyen als ein weiteres Indiz gewertet worden, dass Deutschlands Außen- und Sicherheitspolitik eine klare Linie vermissen lasse und einer strategischen Erosion unterliege. ${ }^{2}$ In diesem Beitrag argumentieren wir, dass es sich aber weniger um eine wirklich neue Entwicklung handelt: Vielmehr folgt die deutsche Außen- und Sicherheitspolitik einem durchaus bekannten Muster von Entscheidungsträgheit, das sich aus historisch-normativen und institutionellen Zwängen ergibt. Neu ist weniger eine Strategieunfähigkeit deutscher Politik, sondern vielmehr die sich wandelnden Bedingungen sicherheitspolitischen Handelns und der sich daraus ergebende Anpassungsdruck auf die Sicherheitsarchitektur, die Deutschland vor größere Herausforderungen stellt als andere westliche und nicht-westliche Staaten. Diese Veränderungen lassen sich analytisch mit dem gängigen handlungsorientierten Verständnis von strategischer Kultur allein nicht fassen. Die wertorientierte und vor allem die bedeutungsorientierte Dimension von Kultur

* Dr. Christopher Daase ist Professor für Internationale Organisationen an der Goethe-Universität Frankfurt und Programmbereichsleiter an der Hessischen Stiftung Friedens- und Konfliktforschung, Kontakt: daase@normativeorders. net. Julian Junk, M.A., ist wissenschaftlicher Mitarbeiter im Forschungsprojekt „Sicherheitskultur im Wandel“, Goethe-Universität Frankfurt a. M., Kontakt: junk@soz.uni-frankfurt.de.

1 Dieser Beitrag profitierte von Diskussionen mit dem Autorenteam des Buchprojekts „Strategic Cultures in Europe“, das vom Sozialwissenschaftlichen Institut der Bundeswehr (SOWI) herausgegeben wird. Eine abgewandelte, englische Version der empirischen Teile dieses Beitrags wird dort erscheinen. Siehe auch den Beitrag von Giegerich und Jonas in diesem Heft.

2 Harald Müller, ,Ein Desaster. Deutschland und der Fall Libyen. Wie sich Deutschland moralisch und außenpolitisch in die Isolation manövrierte', HSFK Standpunkt, Nr. 2, 2011; Hanns W. Maull, 'Deutsche Außenpolitik: Orientierungslos', Zeitschrift für Politikwissenschaft, Bd. 21, Nr. 1, 2011. müssen bei der Einschätzung der Strategiefähigkeit eines Staates mit bedacht werden.

\section{Die Genese von Sicherheitsstrategien als kulturelle Praxis}

Es steht außer Frage, dass sich die Anforderungen an die Außen- und Sicherheitspolitik in den letzten Jahrzehnten massiv gewandelt haben. Die klassischen Unterscheidungen zwischen innerer und äußerer, ziviler und militärischer sowie nationaler und internationaler Sicherheit lösen sich auf. In immer mehr Politikfeldern werden sicherheitspolitische Bedrohungen wahrgenommen (von Klimawandel über Pandemien bis hin zu Wirtschaftskrisen) und Staaten sehen sich nicht mehr nur klar definierten Bedrohungen ausgesetzt, sondern werden von Seiten der Gesellschaft auch für die Reduzierung von weitaus diffuseren Gefahren und Risiken verantwortlich gemacht. ${ }^{3}$ Eine kulturwissenschaftliche Perspektive auf Außen- und Sicherheitspolitik kann zeigen, wie sich das Wechselspiel zwischen gesellschaftlich formulierten Problemlösungsanforderungen und staatlichen Problemlösungsfähigkeiten verändert und welche Folgen das Auseinanderfallen gesellschaftlicher und staatlicher Forderungen und Fähigkeiten hat. ${ }^{4}$ Sowohl für das analytische Verständnis als auch für die politische Weiterentwicklung von Sicherheitspolitik ist der Kulturbegriff deshalb wichtig, weil er die sozialen Praktiken von Individuen und Gesellschaften in Sicherheitsfragen mit einbezieht und Sicherheitspolitik aus dem Korsett angeblich unkontroverser "nationaler Interessen“ befreit. Für eine wirksame, d.h. nachhaltig effektive und legitime Genese von Sicherheitsstrategien in Demokratien ist die gesellschaftliche Debatte zentral.

Das Verhältnis von Kultur und Sicherheitspolitik ist allerdings kein neues Forschungsfeld. ${ }^{5}$ Bereits in den 1960er und 1970er Jahren befasste man sich mit dem Begriff der politischen Kultur.

3 Christopher Daase, Der erweiterte Sicherheitsbegriff - Working Paper 1/2010 des Projekts "Sicherheitskultur im Wandel" (URL: http://www.sicherheitskultur.org/fileadmin/files/WorkingPapers/01-Daase.pdf) (Frankfurt a.M.: Goethe-Universität Frankfurt, 2010).

4 Christopher Daase, ,Sicherheitskultur - Ein Konzept zur interdisziplinären Erforschung politischen und sozialen Wandels', Sicherheit und Frieden, Bd. 29, Nr. 2, 2011.

5 Christopher Daase, Julian Junk und Valentin Rauer, Der sicherheitskulturelle Perspektivenwechsel. Von der politischen und strategischen Kultur zur Sicherheitskultur (Beitrag zur Autorenkonferenz "Sicherheitskultur, 12. Juni 2012; erscheint in einem gleichnamigen Band im VS Verlag, Witten-Herdecke, 2012). 
Kultur wurde hier als Wertorientierung aufgefasst, die politische Einstellungen von Individuen gegenüber politischen Sachverhalten prädisponiert. ${ }^{6}$ Erst Ende der 1980er Jahre und vor allem in den 1990er Jahren wandte man sich der Perspektive der strategischen Kultur zu. Der Kulturbegriff wurde dabei jedoch handlungstheoretisch restriktiv ausgelegt, wodurch das Konzept einen problemlösungsorientierten Fokus erhielt und auf sicherheitspolitische Eliten- und Entscheidungsträger enggeführt wurde. Strategische Kultur wurde als ein Symbolsystem aufgefasst, das einen klaren Handlungsrahmen für die sogenannte Strategic Community bei großen strategischen Entscheidungen, insbesondere über den Einsatz und die Effizienz militärischer Mittel in der internationalen Politik, bietet. ${ }^{7}$ Die Klammer zwischen Öffentlichkeit (in der Forschung zu politischer Kultur) und Elitenforschung (in der Forschung zu strategischer Kultur) fehlte. Erst durch das in den letzten Jahren etablierte Konzept der Sicherheitskultur gelingt dieser Brückenschlag, indem ein bedeutungsorientierter Kulturbegriff ergänzt wurde. ${ }^{8}$ Darauf aufbauend schlagen wir vor, auch bei der Analyse strategischer Kulturen den handlungstheoretischen Kulturbegriff durch einen wert- und bedeutungsorientierten Kulturbegriff zu ergänzen.

Im Folgenden soll anhand der strategischen Kultur in Deutschland diese integrative Perspektive illustriert werden, um den $\mathrm{Zu}$ sammenhang individueller, gesellschaftlicher und staatlicher An- und Herausforderungen im Bereich der Außen- und Sicherheitspolitik deutlich zu machen. Die Operationalisierung erfolgt in Anlehnung an die vier von Alexandra Jonas und Nicolai von Ondarza vorgeschlagenen Analysedimensionen strategischer Kultur: ${ }^{9}$ erstens, das Ambitionsniveau deutscher Außen- und Sicherheitspolitik; zweitens, der Handlungsspielraum der Exekutive; drittens, außenpolitische Traditionen und außenpolitische Orientierung; und viertens, Einstellung zum Einsatz militärischer Instrumente. Diese vier Dimension sind die Grundlage für die folgenden vier Kapitel, welche thesenhaft zugespitzt Teilaspekte der Genese deutscher Sicherheitspolitik beleuchten, dabei aber die Analyseebene der Bevölkerung und die von ihr geäußerten Sicherheitsanforderungen einbeziehen und so ein umfassendes

6 Oscar W. Gabriel, ,Politische Kultur‘, in Viktoria Kaina und Andrea Römmel (Hrsg.), Politische Soziologie. Ein Studienbuch (Wiesbaden: VS Verlag, 2009), S. 22. Siehe auch: Gabriel A. Almond und Sidney Verba, The Civic CulturePolitical Attitudies and Democracy in Five Nations (Princeton, NJ: Princeton University Press, 1965), S. 13.

7 Alistair Iain Johnston, Cultural Realism: Strategic Culture and Grand Strategy in Chinese History (Princeton, NJ: Princeton University Press, 1998), S. 36. Vergleiche auch die Definition von Strategie als „Lehre von Handeln und Gegenhandeln" in Herfried Münkler und Felix Wassermann, ,Von strategischer Vulnerabilität zu strategischer Resilienz: Die Herausforderungen zukünftiger Sicherheitsforschung und Sicherheitspolitik.', in Lars Gerhold und Jochen Schiller (Hrsg.), Perspektiven der Sicherheitsforschung (Frankfurt a.M.: Peter Lang, 2012), S. 82.

8 Christopher Daase, Julian Junk und Valentin Rauer, Der sicherheitskulturelle Perspektivenwechsel. Von der politischen und strategischen Kultur zur Sicherheitskultur; Christopher Daase, 'Sicherheitskultur als interdisziplinäres Forschungsprogramm', in Christopher Daase, Philipp Offermann und Valentin Rauer (Hrsg.), Sicherheitskultur - Soziale und Politische Praktiken der Gefahrenabwehr (Frankfurt a.M.: Campus, 2012). Siehe zusammenfassend für die verschiedenen Kulturbegriffe: Andreas Reckwitz, ,Die Kontingenzperspektive der „Kultur“. Kulturbegriffe, Kulturtheorien und das kulturwissenschaftliche Forschungsprogramm", in Friedrich Jaeger und Jörn Rüsen (Hrsg.), Handbuch der Kulturwissenschaften, Band III: Themen und Tendenzen (Stuttgart/Weimar: J.B. Metzler, 2004); Valentin Rauer, 'Von der Schuldkultur zur Sicherheitskultur. Eine begriffsgeschichtliche Analyse 1986-2010', Sicherheit und Frieden, Bd. 29, Nr. 2, 2011.

9 Alexandra Jonas und Nicolai von Ondarza, Chancen und Hindernisse für die europäische Streitkräfteintegration - Grundlegende Aspekte deutscher, französischer und britischer Sicherheits- und Verteidigungspolitik im Vergleich (Wiesbaden: VSVerlag, 2010); diese vier Dimensionen sind für vergleichende Analysen angelegt. Siehe auch Giegerich und Jonas in diesem Heft.
Bild der strategischen Kultur in der deutschen Außen- und Sicherheitspolitik bieten.

\section{Zwischen Rhetorik und Praxis - Deutschlands verspätete Sicherheitspolitik}

Hinsichtlich des Ambitionsniveaus der deutschen Außen- und Sicherheitspolitik auf einem Kontinuum zwischen passiver Indifferenz und aktivem internationalen Gestaltungswillen würde ein handlungstheoretisch verengter Begriff strategischer Kultur lediglich die strategischen Fähigkeiten der Strategic Community als Maßstab für „Ambition” heranziehen. Allerdings sind auch die artikulierten gesellschaftlichen Erwartungen für die Ambitionen der deutschen Außen- und Sicherheitspolitik entscheidend. Diese gesellschaftlichen Erwartungen werden gerade in ihrer offensichtlichen Diskrepanz zu den staatlichen und internationalen Problemlösungsfähigkeiten wirksam. Denn sie setzen einerseits die Regierung unter Handlungsdruck, insofern Sicherheitsleistungen nicht nur für die eigene Bevölkerung, sondern auch für Menschen in anderen Teilen der Welt (etwa bei humanitären Krisen) eingefordert werden, sie setzen aber andererseits auch dem Regierungshandeln Grenzen, indem hohe Ausgaben für die Erbringung dieser Sicherheitsleistungen abgelehnt und Interventionen, die über rein humanitäre Einsätze hinausgehen, starker Kritik unterzogen werden.

Aus diesem Grund sind in der Vergangenheit die dem Sicherheitsund Verteidigungsapparat zugewiesenen Mittel relativ zum Bundeshaushalt gesunken, und dieser Effekt wird sich durch die Folgen der Finanz- und Staatsschuldenkrise eher noch verstärken. Auch im Vergleich zu den militärischen Ausgaben der engsten NATO-Verbündeten fällt der deutsche Verteidigungsetat deutlich ab: So wendete Deutschland im Jahr 2011 1,3 Prozent seiner Wirtschaftsleistung für militärische Ausgaben auf, während es in Großbritannien 2,6 Prozent, in Frankreich 2,3 Prozent und selbst in Italien noch 1,6 Prozent waren - von den USA mit 4,7 Prozent ganz zu schweigen. ${ }^{10}$ Der NATO-Durchschnitt lag im Jahr 2010 bei 3,3 Prozent. ${ }^{11}$ Während also die finanziellen Aufwendungen stagnierten oder sanken, wuchsen die national wie international formulierten Erwartungen an die deutschen Entscheidungsträger beständig - auch in ihrer Widersprüchlichkeit: Wünsche nach einer stärker an humanitären Prinzipien ausgerichtete Außen- und Sicherheitspolitik, pazifistische Reflexe, aber auch Forderungen nach einer festen transatlantischen Bündnisverankerung als berechenbare und verlässliche europäische Mittelmacht, als zurückhaltender Hegemon in der Mitte Europas, ${ }^{12}$ stellen kaum zu befriedigende Anforderungen dar.

Die Bundesregierung reagierte auf diesen Anpassungsdruck mit Vorschlägen zum Umbau der deutschen Sicherheitsarchitektur,

10 SIPRI, Military Expenditure Database (URL: <http://www.sipri.org/databases/ milex/>), 2011; allgemein macht sich die Finanz- und Staatsschuldenkrise in den Verteidigungshaushalten der europäischen NATO-Mitglieder stark bemerkbar. So wurden sie zwischen 2008 und 2010 teilweise bis zu zehn Prozent gekürzt: siehe IISS, The Military Balance 2012. The Annual Assessment of Global Military Capabilities and Defence Economics (London: International Institute for Strategic Studies, 2012).

11 NATO, Financial and Economic Data Relating to NATO Defence - Communique PR/CP(2011)027 (Brussels: NATO, 10 March 2011).

12 William E. Paterson, ,The Reluctant Hegemon?‘, Journal of Common Market Studies, Bd. 49, Nr. 1, 2011. 
wie sie beispielsweise im Weißbuch 2006 festgehalten sind. ${ }^{13}$ Darin wird die Erweiterung des Sicherheitsbegriffs zu einer der Leitlinien erhoben und ein System vernetzter Sicherheit propagiert. Mehrere konkrete Reforminitiativen schlossen sich an. So empfahl der Bericht der Strukturkommission der Bundeswehr, der sogenannte Weise-Bericht, ${ }^{14}$ eine deutliche Verkleinerung der Bundeswehr auf eine Truppengröße von 185.000 bei einer gleichzeitigen Steigerung der Einsatztruppenstärke, um den multilateralen Verpflichtungen nachkommen zu können. Dazu gehören die rotierende Beteiligung an der NATO Response Force (für Deutschland bedeutet dies, bis zu 15.000 Soldaten und Soldatinnen kurzfristig einsatzfähig zu halten), an den EU Battlegroups und den EU Headline Goals (bis zu 18.000 Soldaten, wobei im Kern pro Battlegroup 1.500 Soldaten eingeplant werden) sowie am UN Standby Arrangements System (nochmals ca. 1.000 Soldaten). ${ }^{15}$ Durch den Bundestag mandatiert sind aktuell 10.240 Soldaten, wobei lediglich 6.255 tatsächlich im Einsatz sind. ${ }^{16}$ Der Weise-Bericht fand seine Konkretisierung im sogenannten Otremba-Bericht zur Neuausrichtung der Bundeswehr ${ }^{17}$ und seine Entsprechung für die Sicherheitsbehörden des Bundes im sogenannten Werthebach-Bericht. ${ }^{18}$ Weitere Reaktionen auf den Anpassungsdruck waren unter anderem die Gründung eines Gemeinsamen Terrorabwehrzentrums (GTAZ) und eines Nationalen Cyber-Abwehrzentrums (NCAZ).

Wiewohl diese Reformliste auf den ersten Blick beeindruckend anmutet, verbleiben vier strukturelle Reformhindernisse: ${ }^{19}$ eine zögerliche Haltung zu einer institutionellen Flexibilisierung, die Fortschreibung der Fragmentierung anstelle der Schaffung nachhaltiger Netzwerkstrukturen, ein Fokus auf die Exekutive und schwer zu ändernde traditionelle Denk- und Handlungsmuster auf Seiten der Entscheidungsträger und der Verwaltung. So zogen sowohl der Werthebach- als auch der Otremba-Bericht starke Kritik auf sich und führten bislang nur zu einer vorsichtigen Restrukturierung innerhalb der alten ministeriellen Einfluss-

13 BMVG, Weißbuch 2006 zur Sicherheitspolitik Deutschlands und zur Zukunft der Bundeswehr (Berlin: Bundesministerium der Verteidigung, 2006); Alexandra Jonas, 'Bis an die Grenzen der Vorstellungskraft? Sicherheitspolitische Bedrohungsanalysen', in Alexandra Jonas und Nicolai von Ondarza (Hrsg.), Chancen und Hindernisse für die europäische Streitkräfteintegration - Grundlegende Aspekte deutscher, französischer und britischer Sicherheits- und Verteidigungspolitik im Vergleich (Wiesbaden: VS Verlag, 2010).

14 BMVG, Bericht der Strukturkommission der Bundeswehr, Oktober 2010 - Vom Einsatz her denken: Konzentration, Flexibilität, Effizienz („Weise-Bericht”) (Berlin: BMVG, 2010).

15 Siehe Nicolai von Ondarza, ,Verfechter eines wirksamen Multilateralismus? Sicherheits- und Verteidigungspolitik auf der internationalen Ebene', in Alexandra Jonas und Nicolai von Ondarza (Hrsg.), Chancen und Hindernisse für die europäische Streitkräfteintegration - Grundlegende Aspekte deutscher, französischer und britischer Sicherheits- und Verteidigungspolitik im Vergleich (Wiesbaden: VS Verlag, 2010b); BMVG, Weißbuch 2006 zur Sicherheitspolitik Deutschlands und zur Zukunft der Bundeswehr; BMVG, Bericht der Strukturkommission der Bundeswehr, Oktober 2010 - Vom Einsatz her denken: Konzentration, Flexibilität, Effizienz (,Weise-Bericht").

16 Aktuelle Zahlen vom 11. Juli 2012; siehe www.bundeswehr.de. Von den aktuell im Einsatz befindlichen Truppen, sind in Afghanistan im Rahmen des ISAFEinsatzes 4.931, im Kosovo im Rahmen von KFOR 710, am Horn von Afrika im Rahmen von Atalanta 320 und im Rahmen von UNIFIL vor dem Libanon 219 Soldaten stationiert.

17 BMVG, Konzentration und Verantwortung - Die prozessorientierte Neuausrichtung der Bundeswehr („Otremba-Bericht” vom 7. Februar 2011) (Berlin: BMVG, 2011). 18 Ebenda.

19 Christopher Daase und Julian Junk, ,Netzwerke der Sicherheit. Überlegungen zum Umbau demokratischer Sicherheitspolitik', Sicherheit und Frieden, Bd. 30 , Nr. 2, 2012; Bundesregierung, 2. Bericht der Bundesregierung über die Umsetzung des Aktionsplans „Zivile Krisenprävention, Konfliktlösung und Friedenskonsolidierung" (Berlin: Bundesregierung, 2008). bereiche. ${ }^{20}$ So wird die Trennung von ziviler und militärischer Sicherheit reproduziert, wie nicht zuletzt das bei aller Betonung nicht-traditioneller Aspekte stark militärisch ausgerichtete Konzept der vernetzten Sicherheit zeigt. ${ }^{21}$ Dabei werden zwar intra-organisationale Effizienzgewinne erzielt, wie etwa durch die Einrichtung eines Einsatzführungsstabs im Bundesverteidigungsministerium, inter-organisationale Strukturen (wie die dringend verbesserungswürdige interministerielle Koordination $^{22}$ ) werden aber vernachlässigt. Es bleibt eine starke Trennung zwischen politischer, strategischer und operativer Ebene erhalten. Ein Grund dafür, warum sich Deutschland beispielsweise in Afghanistan mit der Aufgabenerfüllung im Rahmen von ISAF schwer tat, ist das nach wie vor im deutschen Sicherheitsapparat tendenziell vorherrschende defensive Denkmuster der Landesverteidigung, ${ }^{23}$ das auch bei den ersten Erfahrungen mit friedenserhaltenden (nicht friedenschaffenden) Bundeswehreinsätzen auf dem Balkan nicht grundlegend verändert wurde. ${ }^{24}$ Diese Haltung verbindet sich mit dem traditionellen, seit der Gründung der Bundeswehr bestehenden Bemühen, jeglichen Anschein von hegemonialem Großmachtstreben auf der Basis von Wirtschaftsmacht zu vermeiden. ${ }^{25}$ Die „Kultur der militärischen Zurückhaltung " wird dadurch weiter gestärkt. ${ }^{26}$

Dies alles mag erklären, warum die deutsche Außen- und Sicherheitspolitik zwar auf den Handlungsdruck, der auf sie sowohl von der eigenen Gesellschaft als auch der internationalen Gemeinschaft ausgeübt wird, reagiert, sie aber dennoch zu Passivität und zur Innenschau neigt. Während die Regierung etwa auf ökonomischem und umweltpolitischem Gebiet wesentlich proaktiver auftritt, ${ }^{27}$ bleibt es bei dem Befund für die Sicherheitspolitik, dass Deutschland ein „verspäteter" strategischer Akteur ist und nicht selten den Eindruck erweckt, seinen eigenen Ambitionen nicht gerecht zu werden.

\section{Gezähmte Exekutive: außenpolitische Handlungsspielräume deutscher Regierungen}

Zweites zentrales Kriterium für die strategische Kultur eines Landes ist die sicherheitspolitische Handlungsfähigkeit der Exekuti-

20 Siehe unter anderem zur Kritik am Werthebach-Bericht: ZEIT, Fusion von Bundespolizei und BKA abgesagt (URL: http://www.zeit.de/politik/deutschland/2011-03/polizei-reform-bka), 15. März 2009).

21 Bundesregierung, 2. Bericht der Bundesregierung über die Umsetzung des Aktionsplans „Zivile Krisenprävention, Konfliktlösung und Friedenskonsolidierung“; Martin Neujahr, 'Vernetzte Operationsführung und das neue operative Umfeld: gesteigerte Einsatzwirksamkeit durch verbesserte Führungsfähigkeit', in Heiko Borchert (Hrsg.), Vernetzte Sicherheit. Leitidee der Sicherheitspolitik im 21. Jahrhundert (Hamburg: Mittler \& Sohn, 2004); Timo Noetzel, ,The German politics of war: Kunduz and the war in Afghanistan', International Affairs, Bd. 87, Nr. 2, 2011, S. 403.

22 Timo Noetzel, ,Germany‘s Small War in Afghanistan: Military Learning amid Politico-strategic Inertia', Contemporary Security Policy, Bd. 31, Nr. 3, 2010 , S. 499.

23 Benjamin Schreer, ,Political Constraints: Germany and Counterinsurgency Security Challenges, Bd. 6, Nr. 1, 2010, S. 97-100.

24 Timo Noetzel, ,Germany‘s Small War in Afghanistan: Military Learning amid Politico-strategic Inertia', S. 486-508.

25 Hans Kundnani, ,Germany as a Geo-economic Power', Washington Quarterly, Bd. 34, Nr. 3, 2011

26 Kerry Longhurst, Germany and the Use of Force - the Evolution of German Security Policy 1990-2003 (Manchester: Manchester University Press, 2004), S. 130.

27 Tanja Brühl, ,Umweltpolitik', in Siegmar Schmidt, Gunther Hellmann und Reinhard Wolf (Hrsg.), Handbuch zur deutschen Außenpolitik (Wiesbaden: VS Verlag, 2007); Michael Staack, 'Deutschland als Wirtschaftsmacht', in ebenda; im Falle des wirtschaftlichen Gewichts Deutschlands nicht ohne Kritik: Erik Jones, ,Merkel‘s Folly‘, Survival, Bd. 52, Nr. 3, 2010. 
ve. Auch wenn die deutsche Bundesregierung der entscheidende Akteur in der Außen- und Sicherheitspolitik bleibt, so ist sie im Vergleich zu den engsten europäischen sicherheitspolitischen Verbündeten wie Frankreich und Großbritannien stark eingeschränkt - insbesondere durch drei Faktoren:

Erstens durch den starken Parlamentsvorbehalt bei Mandatierung und Mandatsverlängerungen von Auslandseinsätzen der Bundeswehr. ${ }^{28}$ Die Balance zwischen Regierung und Bundestag wurde im Parlamentsbeteiligungsgesetz im Jahr 2005 geregelt. Lediglich bei „Gefahr im Verzug“ kann die Bundesregierung ohne Zustimmung des Bundestages Truppen entsenden, allerdings muss sie unverzüglich das Mandat einholen, ansonsten hat der Bundestag ein (nicht unumstrittenes) Rückholrecht. ${ }^{29}$ Nicht umsonst wird die Bundeswehr häufig als „Parlamentsarmee“ bezeichnet. Der Bundesregierung kam allerdings bislang zugute, dass ihre Anträge in diesem Bereich nur in operationalen Details, aber nicht grundsätzlich verändert werden dürfen - alternativ bleibt lediglich die Ablehnung. Darüber hinaus stellten insbesondere die Mandatsverlängerungen lange Zeit eine weitgehend entpolitisierte Routine dar - was sich erst mit der weiter unten beschriebenen Kontroverse um den Afghanistan-Einsatz änderte.

Eine Ausnahme von der institutionell verankerten parlamentarischen Kontrolle im außen- und sicherheitspolitischen Bereich bildet der Bundessicherheitsrat, der von der Bundeskanzlerin und den zuständigen Bundesministern gebildet wird. Ein Versuch von Seiten der CDU/CSU-Fraktion, dem Bundessicherheitsrat zu einem breiteren Mandat zur Koordinierung der Außen- und Sicherheitspolitik der Bundesregierung zu verhelfen, scheiterte, als dieser Plan in Verbindung mit dem traditionell umstrittenen Thema von Bundeswehreinsätzen im Inneren gebracht wurde. ${ }^{30}$ So blieb der Bundessicherheitsrat eine Institution, die mehr oder weniger im Verborgenen (und mehr oder weniger effektiv) deutsche Waffenexporte kontrolliert.

Zweitens hat die öffentliche Meinung einen immer stärkeren Einfluss auf außen- und sicherheitspolitische Entscheidungen gewonnen - nicht zuletzt angesichts der Bedeutung, die die Ablehnung des Irakkriegs bei der Bundestagswahl 2002 gespielt hat. In Verbindung mit dem oben beschriebenen Parlamentsvorbehalt, der in der Öffentlichkeit eine große Zustimmung erfährt, ${ }^{31}$ werden Entscheidungen zu Auslandseinsätzen zunehmend breit bis in die Wahlkreise hinein - diskutiert.

Drittens ist die Verfassungsgerichtsbarkeit in Deutschland auch im Bereich der Außen- und Sicherheitspolitik einflussreich. Die

28 Rafael Biermann, ,Der Deutsche Bundestag und die Auslandseinsätze der Bundeswehr. Zur Gratwanderung zwischen exekutiver Prärogative und legislativer Mitwirkung', Zeitschrift für Parlamentsfragen, Bd. 35, Nr. 4, 2004.

29 Andreas Gilch, Das Parlamentsbeteiligungsgesetz: die Auslandsentsendung der Bundeswehr und deren verfahrensrechtliche Ausgestaltung (URL: http://opus.bibliothek.uni-wuerzburg.de/volltexte/2005/1441/) (Würzburg: Universität Würzburg OPUS, S. 147-157; zitiert in Nicolai von Ondarza, ,Eine Frage der Flexibilität. Rechtliche Rahmenbedingungen für den Streitkräfteeinsatz', S. 67. Siehe auch: Nicolai von Ondarza, Legitimatoren ohne Einfluss? Nationale Parlamente in Entscheidungsprozessen zu militärischen $E U$ - und VN-Operationen im Vergleich (Baden-Baden: Nomos, 2012), S. 159-169.

30 Timo Noetzel, ,Germany's Small War in Afghanistan: Military Learning amid Politico-strategic Inertia', S. 499.

31 Heiko Biehl, ,Strategische Kulturen im Meinungsbild der europäischen Bevölkerungen', in Heiko Biehl, Rüdiger Fiebig, Bastian Giegerich, Jörg Jacobs und Alexandra Jonas (Hrsg.), Strategische Kulturen in Europa. Die Bürger Europas und ihre Streitkräfte. Ergebnisse der Bevölkerungsbefragungen in acht europäischen Ländern 2010 des Sozialwissenschaftlichen Instituts der Bundeswehr. Forschungsbericht 96. (Strausberg: SOWI, 2011), S. 43-45. große Wende hin zur Ermöglichung von Out-of-area-Einsätzen in den 1990er Jahren wurde maßgeblich vom Bundesverfassungsgericht mitgestaltet, stellte es doch Kriterien auf (VN-Mandat und Parlamentsvorbehalt), unter denen diese Einsätze trotz des Verbots in Artikel 87a des Grundgesetzes möglich sind. Das Gericht reagierte damit auf die neue Bündniswirklichkeit nach der Wiedervereinigung und dem Ende des Kalten Krieges und ebnete den Weg zum Parlamentsbeteiligungsgesetz. Ohne diese Entscheidung des Bundesverfassungsgerichts wäre eine Beteiligung der deutschen Bundeswehr beim Luftangriff auf Serbien während der Kosovokrise 1999 nicht möglich gewesen.

Diese institutionellen Checks and Balances führen zu einer auf Konsens ausgerichteten sicherheitspolitischen Debatte und einem System der Entscheidungsfindung, das politischer Kontinuität verpflichtet ist. ${ }^{32}$ Zwar hat dieses System in den letzten Jahren durch kontroverse Entscheidungen im Zuge des Afghanistan-Einsatzes einige Risse bekommen; gleichwohl bleibt die Regierung auf die Unterstützung von Parlament, Öffentlichkeit und Verfassungsgerichtsbarkeit angewiesen. Die vielfältigen Abstimmungsprozesse zwischen diesen Institutionen lassen die Entscheidungen in der Außen- und Sicherheitspolitik relativ schwerfällig erscheinen, mitunter sogar erratisch; aber sie lassen keine Beliebigkeit im Sinne eines strategischen Vakuums erkennen.

\section{Multilateralismus und Westbindung: außenpolitische Traditionen}

Laut Jonas und von Ondarza ${ }^{33}$ spielt zur Einordnung der strategischen Kultur eines europäischen Landes das Kontinuum zwischen einer EU-Orientierung und einer NATO- bzw. Transatlantikorientierung eine wichtige Rolle. Wiewohl Deutschland sicherlich zu den Staaten Europas gehört, die hier eher eine Mittlerposition einnehmen und auch während der Kanzlerschaft Angela Merkels darauf bedacht ist, im außen- und sicherheitspolitischen Bereich die EU und die NATO nicht gegeneinander auszuspielen, so finden sich in den offiziellen Dokumenten der Bundesregierung doch einige feine Unterscheidungen: Die NATO wird hier weiterhin als das Instrument für robuste militärische Einsätze gesehen, während der komparative Vorteil der EU in ihren breiten, auch zivile Akteure und Instrumente umfassenden Fähigkeiten liegt. Es komme daher darauf an, „die unterschiedlichen Profile, Kompetenzen und Stärken von EU und NATO mit dem Ziel der Komplementarität auszubauen und möglichst effizient zu nutzen [...] Daneben steht aber auch das Ziel, die Europäische Union zur autonomen Planung und Führung von ESVP-Operationen zu befähigen. "34 Obgleich es offensichtlich eine klare Aufgabenteilung gibt und die deutsche Bundesregierung, etwa im Gegensatz zu Großbritannien, den EU-Arm stärken möchte, bleibt sie doch beiden Organisationen zugewandt und ihrer Komplementarität verpflichtet.

Eine Spannung zwischen europäischer und transatlantischer Ausrichtung lässt sich somit auf der Ebene der Doktrin nicht er-

32 Lars Colschen, Deutsche Außenpolitik (Stuttgart: UTB, 2010), S. 386.

33 Alexandra Jonas und Nicolai von Ondarza, Chancen und Hindernisse für die europäische Streitkräfteintegration - Grundlegende Aspekte deutscher, französischer und britischer Sicherheits- und Verteidigungspolitik im Vergleich, S. 23-24.

34 BMVG, Weißbuch 2006 zur Sicherheitspolitik Deutschlands und zur Zukunft der Bundeswehr, S. 48-49. 
kennen, wohl aber institutionell innerhalb der Bundesregierung anhand der relativ starren Aufgabenteilung und einer mangelnden interministeriellen Koordination ausmachen: Während das Kanzleramt sowie die zuständigen Fachressorts wie die Bundesministerien für Finanzen, für Inneres, für Wirtschaft und Technologie oder für die Justiz sich auf Europa konzentrieren, dominiert im Bundesministerium der Verteidigung ein Fokus auf die NATO. Das Auswärtige Amt, eigentlich neben dem Kanzleramt zuständig für die Kohärenz zwischen den Ministerien in diesen Fragen der Außen- und Sicherheitspolitik, scheitert nicht selten an dieser Aufgabe aufgrund des hohen Grads ministerieller Autonomie.

Umstrittener als die inner-westliche Ausrichtung zur EU oder zur NATO war jedoch immer die Ost-West-Kontroverse in der deutschen Nachkriegsgeschichte - siehe Willy Brandts Ostpolitik oder das zur Schau gestellte enge Verhältnis mit russischen Staatslenkern unter den Kanzlerschaften von Helmut Kohl und Gerhard Schröder. Gerade bei den osteuropäischen Nachbarstaaten werden solche Annäherungsversuche kritisch gesehen, wie zuletzt der Bau der North-Stream-Pipeline durch die Ostsee. Allerdings ließ die Bundesregierung nie wirklich einen Zweifel an ihrer Westorientierung aufkommen. Dennoch hat erst Merkels distanzierteres Verhältnis zu Putin zahlreiche Skeptiker besänftigt.

Unstrittig ist aber in jedem Fall das Bekenntnis zum Multilateralismus. Auch wenn einige Kommentatoren die Entscheidungen zu Libyen im Jahr 2011 oder vorher zum Irak 2002, Stellungnahmen zum Rückzug von US-Nuklearwaffen aus deutschen Standorten 2010 oder das negative Amerikabild der Deutschen zu Zeiten der Bush-Administration ${ }^{35}$ als eine Tendenz zum Unilateralismus werteten, bleibt die grundsätzliche Ausrichtung deutscher Außen- und Sicherheitspolitik multilateral - wenngleich „strategisch multilateral“36 oder „selektiv multilateral.“37 Die stärkere Artikulation nationaler Interessen vor allem im multilateralen Rahmen der Vereinten Nationen, wie sie Frankreich und Großbritannien schon lange praktizieren, wird zumindest von internationalen Beobachtern meist als „Normalisierung“ bezeichnet. ${ }^{38}$ In der deutschen Debatte ist allerdings schon der Begriff „nationales Interesse“ umstritten, weil er suggeriert, dass die deutschen Interessen von den europäischen oder globalen Interessen abweichen könnten - eine Vorstellung, die dem uneingeschränkt positiven Verständnis von Multilateralismus zu widersprechen scheint.

Zur strategischen Kultur der Bundesrepublik gehört somit eine starke, mitunter unkritische, Befürwortung multilateraler Institutionen, wobei die öffentliche Zustimmung mit dem Grad der Inklusivität steigt: Die UNO erfreut sich größeren Zuspruchs als die NATO.

35 Thomas Bulmahn, Rüdiger Fiebig, Stefanie Greif, Alexandra Jonas, Wolfgang Sender und Victoria Wieninger, Sicherheits- und verteidigungspolitisches Meinungsklima in der Bundesrepublik Deutschland Ergebnisse der Bevölkerungsbefragung 2007 des Sozialwissenschaftlichen Instituts der Bundeswehr - Forschungsbericht 86 (Strausberg: SOWI, 2008), S. 41.

36 Helga Haftendorn, Deutsche Außenpolitik zwischen Selbstbeschränkung und Selbstbehauptung (Stuttgart: Deutsche Verlags-Anstalt, 2001), S. 15.

37 Hans Kundnani, ,Germany as a Geo-economic Power', S. 35-39.

38 Simon Bulmer und William E. Paterson, ,Germany and the European Union: from 'tamed power' to normalized power?', International Affairs, Bd. 86, Nr. 5 , 2010; Regina Karp, 'Germany: A “Normal” Global Actor?', German Politics, Bd. 18 , Nr. 1, 2009.

\section{Zögerliche Mittelmacht: die militärischen Instrumente deutscher Außen- und Sicherheitspolitik}

Eine letzte Dimension strategischer Kultur ist die Bereitschaft, militärische Mittel für politische Zwecke einzusetzen. Wie schnell wird in der deutschen Außen- und Sicherheitspolitik auf militärische Lösungen gesetzt und wie bereitwillig werden Truppenkontingente zur Verfügung gestellt? Bei der Beantwortung dieser Frage kommt es darauf an, ob man den deutschen Militäreinsatz im Kosovo und in Afghanistan als einen Trend zu größerer Bereitschaft ansieht, auch robuste Mandate, also solche, die Kampfhandlungen einschließen, wahrzunehmen, oder ob man die Nicht-Beteiligung an der Libyen-Intervention als Beweis dafür nimmt, dass der Einsatz militärischer Mittel immer noch eher die Ausnahme als die Regel ist.

Nach dem Zweiten Weltkrieg wurde Deutschland als „Wirtschafts-“ und „Zivilmacht" beschrieben - eine Macht in der Mitte Europas, die das Primat des Multilateralismus pflegt, die auch der Souveränitätsübertragung auf supranationaler Ebene aufgeschlossen gegenübersteht und die generell einen eher pazifistischen Reflex aufweist. ${ }^{39}$ Weite Teile der öffentlichen Meinung und der politischen Elite in Deutschland teilen dieses Selbstbild nach wie vor. Sie sehen Deutschland strategisch eher als risikoavers und defensiv. ${ }^{40}$ Der Satz des ehemaligen Bundesverteidigungsministers Peter Struck, dass Deutschland seine Interessen auch am Hindukusch verteidigt, wird eher augenzwinkernd zitiert; und Bundespräsident Horst Köhler trat aufgrund einer Kontroverse um die angebliche Befürwortung des Militäreinsatzes zur Sicherung ökonomischer Interessen zurück, auch wenn dies im Rahmen der Atalanta-Mission am Horn von Afrika längst ein Faktum war. Beides zeigt, dass die deutsche Öffentlichkeit militärischen Instrumenten ausgesprochen skeptisch gegenübersteht: Nur 14 Prozent der Bevölkerung befürworten den Einsatz militärischer Mittel zur Bewältigung von internationalen Krisen und im Konfliktmanagement, 50 Prozent sprechen sich hingegen für wirtschaftliche Instrumente (wie Sanktionen) und gar 83 Prozent für einen Fokus auf rein diplomatische Mittel aus. ${ }^{41}$ Das Institut für Demoskopie Allensbach sieht in diesen Zahlen sogar Tendenzen des Isolationismus. ${ }^{42}$

Allerdings spielt die „Rahmung“ von militärischen Interventionen eine entscheidende Rolle. Denn je nachdem, wie ein Militäreinsatz gerechtfertigt wird, neigt die öffentliche Meinung zu Akzeptanz oder Ablehnung. Humanitäre Gründe und ordnungspolitische Ziele wie Friedenssicherung werden mehrheitlich gebilligt, während nationale Interessen oder wirtschaftliche Zwe-

39 Vergleiche zum Konzept der Zivilmacht: Hanns W. Maull, ,Germany and Japan: the New Civilian Powers', Foreign Affairs, Bd. 69, Nr. 5, 1990.

40 Timo Noetzel und Benjamin Schreer, ,All the Way? The Evolution of German Military Power', International Affairs, Bd. 84, Nr. 2, 2008.

41 Heiko Biehl, ,Strategische Kulturen im Meinungsbild der europäischen Bevölkerungen', S. 46-48. Siehe auch Christian Freuding, Streitkräfte als Instrument deutscher Außen- und Sicherheitspolitik seit Mitte der Neunziger Jahre (Hamburg: Helmut Schmidt Universität, 2007).

42 Elisabeth Noelle und Thomas Petersen, Ein Hauch von Isolationismus Allensbach-Analyse (Frankfurt a.M.: Frankfurter Allgemeine Zeitung, 24. Januar 2007). 
cke weitgehend als illegitim gelten. ${ }^{43}$ Nicht ohne Grund zögerte die Bundesregierung in den Jahren 2007-2011 lange, den Einsatz in Afghanistan als Kriegseinsatz zu bezeichnen. ${ }^{44}$ Denn dies drohte, die bis dahin geltende Rechtfertigung der Intervention als Stabilisierungs- und Wiederaufbaumaßnahme zu konterkarieren ${ }^{45}$ und die Bevölkerung, die dem Einsatz ohnehin skeptisch gegenüberstand, zu alarmieren. Die Befürchtung war gerechtfertigt: Die Bundesregierung hatte nach dem vom deutschen Regionalkommando im Norden Afghanistans angeforderten Luftschlag gegen Tanklastzüge bei Kunduz am 4. September 2009 ihre Sprachregelung nicht aufrechterhalten können. Es war nicht länger zu leugnen, dass auch das deutsche ISAF-Kontingent Teil eines „Krieges“ war. Der ARD-Deutschlandtrend stellte im Mai 2010 fest, dass zu diesem Zeitpunkt bereits über einen längeren Zeitraum bis zu 70 Prozent der Befragten für einen sofortigen Abzug aller deutschen Truppen aus Afghanistan waren.

Wie sehr die traditionell defensiven Denkmuster auch den Alltag der Planung und Durchführung von militärischen Einsätzen prägen, wird deutlich, wenn man sich mit den deutschen Einsatzregeln für Soldaten vertraut macht. Zwar wird jeder multilaterale Einsatz durch international verhandelte Einsatzregeln sogenannte Rules of Engagement - vorgegeben, jedoch lassen diese explizit Spielraum für nationale Befindlichkeiten, um eine Einigung auf dem kleinsten gemeinsamen Nenner zu vermeiden. Im deutschen Fall sind diese nationalen Ausnahmeregelungen besonders ausgeprägt, mit weitgehenden Auswirkungen für die eigene und die multilaterale Einsatzdurchführung. ${ }^{46}$ So bekommen deutsche Soldaten im Einsatz sogenannte „Taschenkarten“, im Fall von Afghanistan vierseitige Merkblätter, die einfach und verständlich sowohl die multilateralen Einsatzregeln und das Mandat als auch die nationalen rechtlichen Vorgaben zur Gewaltanwendung darstellen. ${ }^{47}$ Diese Taschenkarten sind bei jedem Einsatz mitzuführen. In ihrer vergleichsweise restriktiven Ausformulierung spiegelt sich die Hoffnung, dass allein durch militärische Präsenz Stabilität zu erreichen sei und die Konfliktdynamik vor Ort deshalb vernachlässigt werden könne. ${ }^{48}$

Die deutsche Außen- und Sicherheitspolitik ist nach wie vor von einer durchgängig ambivalenten Haltung gegenüber dem Einsatz militärischer Gewalt gekennzeichnet. Sowohl in der Bevölkerung als auch in der politischen Elite werden militärische Maßnahmen allenfalls als Ultima Ratio angesehen, als letztes Mittel, das nicht einfach Teil einer instrumentellen Kosten-Nutzen-Abwägung sein darf. Diese Ambivalenz ist bis in die Streitkräfte hinein wahrzunehmen, deren Einsatzregeln vielfach so restriktiv formuliert

43 Thomas Bulmahn, Rüdiger Fiebig, Stefanie Greif, Alexandra Jonas, Wolfgang Sender und Victoria Wieninger, Sicherheits- und verteidigungspolitisches Meinungsklima in der Bundesrepublik Deutschland Ergebnisse der Bevölkerungsbefragung 2007 des Sozialwissenschaftlichen Instituts der Bundeswehr - Forschungsbericht 86, S. 107; Julian Junk, 'Humanitäre Interventionen als sicherheitskulturelle Praxis', in Christopher Daase, Philipp Offermann und Valentin Rauer (Hrsg.), Sicherheitskultur - Soziale und Politische Praktiken der Gefahrenabwehr (Frankfurt a.M.: Campus, 2012).

44 Nicholas Kulish, German Limits on War Are Facing Reality in Afghanistan, in: The New York Times, 27. Oktober 2009).

45 Benjamin Schreer, ,Political Constraints: Germany and Counterinsurgency“, S. 99.

46 Timo Noetzel, ,Germany‘s Small War in Afghanistan: Military Learning amid Politico-strategic Inertia', S. 492.

47 Jochen Bittner, ,Grundkurs Krieg, in: DIE ZEIT (URL: http://www.zeit. de/2009/33/Buecher-machen-Politik-Taliban)`, 6. August 2009.

48 Dirk Freudenberg, ,Das Spannungsverhältnis im operativen Einsatz: Auftragstaktik, Rules of Engagement und deutsche Strafrechtsordnung', Neue Zeitschrift für Wehrecht, 2007. sind, dass sie im Rahmen multilateraler Interventionen zu Kooperationsproblemen mit den Partnern führen. Das uneinheitliche Bild, das Deutschland im Hinblick auf seine Beteiligung an multilateralen Militärinterventionen abgibt - Beteiligung im Kosovo und in Afghanistan, Ablehnung im Irak und in Libyen ist vor allem auf diese Ambivalenz zurückzuführen. Sie wird auf absehbare Zeit die strategische Kultur Deutschlands prägen.

\section{Schlussfolgerungen}

In allen vier Dimensionen strategischer Kultur - Ambition, Handlungsspielraum, institutionelle Orientierung und Haltung zum Militäreinsatz - lassen sich starke Faktoren identifizieren, welche die deutsche Außen- und Sicherheitspolitik auf eine eher defensive und reaktive, passive und zurückhaltende Position festlegen. Im Hinblick auf außenpolitische Ambitionen hat das Auswärtige Amt zwar unlängst mit dem Konzept der „Gestaltungsmächte“49 selber einen stärkeren globalen Gestaltungswillen und mithin eine aktivere Außen- und Sicherheitspolitik Deutschlands angedeutet; in sicherheitspolitischer Perspektive bleiben jedoch die nationalen Ambitionen moderat und werden vorrangig im Rahmen multilateraler Institutionen kommuniziert. Dabei bleibt der Handlungsspielraum der Bundesregierung dreifach beschränkt: durch ein starkes Parlament, durch eine einflussreiche öffentliche Meinung und durch die Verfassungsgerichtsbarkeit. Sicherheitspolitisches Handeln muss auf unterschiedlichen Ebenen innenpolitisch abgestimmt und abgesichert werden und unterliegt damit langwierigen institutionellen Entscheidungsprozessen. Weiterhin fußt die deutsche Außen- und Sicherheitspolitik auf einer starken Westbindung und einem strategischen Multilateralismus. Dabei haben es die Bundesregierungen immer vermieden, die NATO gegen die Europäische Union auszuspielen. Allerdings kommt es durch eine starke Ressortautonomie in der deutschen Ministerialverwaltung zu einer gewissen Fragmentierung, die gelegentlich zu Inkohärenz zwischen europäischer und transatlantischer Politik führt. Schließlich ist die deutsche strategische Kultur nur dann zu verstehen, wenn man die Ambivalenz gegenüber militärischen Mitteln sowohl in der Bevölkerung als auch der politischen Elite berücksichtigt. Entwicklungen hin zu einer proaktiveren, exekutiv-orientierten, selbstbewussteren und interventionistischeren Außen- und Sicherheitspolitik - von den einen gefordert, von den anderen befürchtet - sind deshalb durch die strategische Kultur enge Grenzen gesetzt. Das heißt aber nicht, dass man in die „Falle der Kontinuität“50 tappen würde. Die Geschwindigkeit von Anpassungs- und Wandlungsprozessen mag langsam sein, organisationale Reformen nicht weit genug reichen oder gar verschleppt werden, aber strategielos, erratisch und gar ohne jede strategische Kultur ist die deutsche Außen- und Sicherheitspolitik sicher nicht.

49 AA, Globalisierung gestalten - Partnerschaften ausbauen - Verantwortung teilen. Konzept der Bundesregierung (Berlin: Auswärtiges Amt, 2012).

50 Eberhard Sandschneider, ,Deutschland: Gestaltungsmacht in der Kontinuitätsfalle', Aus Politik und Zeitgeschichte, Bd. 62, Nr. 10, 2012. 